\title{
Mobile Phone Based Drunk Driving Detection
}

\author{
Jiangpeng Dai $^{* \dagger}$, Jin Teng ${ }^{\dagger}$, Xiaole Bai ${ }^{\dagger}$, Zhaohui Shen ${ }^{\ddagger}$ and Dong Xuan ${ }^{\dagger}$ \\ * School of Computer Sci. \& Engr. \\ Southeast University \\ Nanjing, Jiangsu, China \\ jpdai@seu.edu.cn \\ $\dagger$ Dept. of Computer Sci. and Engr. \\ The Ohio State University \\ Columbus, Ohio, USA \\ \{jpdai, tengj, baixia, xuan\}@cse.ohio-state.edu \\ $\ddagger$ Division of Physical Therapy \\ The Ohio State University \\ Columbus, Ohio, USA \\ shen.119@osu.edu
}

\begin{abstract}
Drunk driving, or officially Driving Under the Influence (DUI) of alcohol, is a major cause of traffic accidents throughout the world. In this paper, we propose a highly efficient system aimed at early detection and alert of dangerous vehicle maneuvers typically related to drunk driving. The entire solution requires only a mobile phone placed in vehicle and with accelerometer and orientation sensor. A program installed on the mobile phone computes accelerations based on sensor readings, and compares them with typical drunk driving patterns extracted from real driving tests. Once any evidence of drunk driving is present, the mobile phone will automatically alert the driver or call the police for help well before accident actually happens. We implement the detection system on Android G1 phone and have it tested with different kinds of driving behaviors. The results show that the system achieves high accuracy and energy efficiency.

Keywords-Drunk Driving Detection, Mobile Phones, Acceleration, Sensors
\end{abstract}

\section{INTRODUCTION}

\section{A. Motivation}

Crashes caused by impairment of alertness in vehicle drivers pose a serious danger to people, not only to drivers themselves but also often to the general public [1]. According to the report of U.S. National Highway Traffic Safety Administration (NHTSA), more than a million people have died in traffic crashes in the United States since 1966. During these tragedies, drunk driving is one of the main causes. The concern related to drunk driving is not only the high crash rate, but also the type of crashes that are most likely to happen. In the last two years, 2007 and 2008, 13,041 and 11,773 alcohol-impaired driving fatalities happened, respectively. Both are $32 \%$ of the total fatalities of that year [2]. During these crashes, ten of thousands of people were killed, and much more people injured. Besides being a great threat to public safety and health, drunk driving also imposes a heavy financial burden on the whole society, especially on the healthcare sector. According to U.S. Central of Disease control (CDC) [3], the annual cost of alcoholrelated crashes totals more than $\$ 51$ billion in 2008. Lee et al. pointed out in their work [4] that the emergency department spends $\$ 4,538$ more on average in treating alcohol-impaired motor vehicle crash victims, especially for patients who are minimally injured, because of their impaired reasoning and blunted sensation.

Despite the fact that drunk driving is a serious problem, its detection has been so far relying on visual observations by patrol officers. Drivers under the influence of alcohol show a marked decline of perception, recognition, and vehicle control, so they tend to make certain types of dangerous maneuvers. The U.S. NHTSA has conducted extensive studies in their effort to help distinguish these maneuvers. During field studies involving hundreds of officers and more than 12,000 enforcement stops, the researchers have identified cues of typical driving behavior for drunk drivers, namely problems in maintaining proper lane position, speed and braking problems, vigilance problems and judgment problems [5]. These are guidelines for patrol officers to stop a suspect drunk driver and give him an alcohol test.

However, relying on visual observation of patrol officers to prevent drunk driving is insufficient. First of all, given the huge mileage of driveways in USA, the number of patrol officers is far from enough to observe and analyze every driver's behaviors. Second, the guidelines of drunk driving patterns are only descriptive and qualitative. Sometimes it is not easy to tell whether a vehicle is performing that exact type of movements or not, especially when it is dark with poor sight range or other obstructions are present in the middle. In fact, U.S. CDC has reported that each year only less than $1 \%$ of the drunk drivers are arrested and more than $99 \%$ of self-alleged drunk driving episodes go unnoticed [3]. It is also imaginable that even a drunk driver is stopped by a patrol officer, the driver may have been on the way for dozens of miles. So it is essential to develop systems actively monitoring drivers' operating situations and alerting of any insecure conditions to prevent accident. It is preferable that the actively monitoring system satisfies the following requirements: a real-time monitoring system with quick response; a reliable system performing accurately; a nonintrusive system and a low cost system.

\section{B. Our Contributions}

In this paper, we propose utilizing mobile phones as the platform for drunk driving detection system development, as they naturally combine the detection and communication functions. To the best of our knowledge, we are the first to do so.

As a self-contained device, mobile phone presents a mature hardware and software environment for the development of active drunk driving monitoring system. The system based on mobile phone can function effectively on its own because mobile phones are highly portable, all necessary components are already integrated therein, and their communication services 
have vast coverage. The minimum requirement for such a mobile phone platform is the presence of simple sensors, e.g., accelerometer and orientation sensor. Currently, many phones, especially smartphones, meet this requirment. They contain multiple types of sensors, including accelerometers and orientation sensors. And their communication module and speakers are naturally good enough for alerting. Such phones are very popular and widely accepted in our society. Over 120 million smartphones were sold in 2008 [6], and their popularity is projected to continuously increase in the near future due to decreasing price. Recently, several leading telecommunication companies such as AT\&T have made available affordable smartphones [7] [8], whose features are similar to those of highend models, in addition to cheaper service plans [9].

We summarize the contributions of this paper as follows.

- We propose utilizing mobile phones as the platform for drunk driving detection. To the best of our knowledge, we are the first to introduce mobile phones in the area of drunk driving detection.

- We design the algorithm for detecting drunk driving in real time using mobile phones. We analyze the drunk driving related behaviors and extract its fundamental cues based on lateral and longitudinal accelerations of vehicle, which are determined by accelerometer and orientation sensor readings in mobile phones.

- We design and implement the drunk driving detection system on mobile phones. The system is reliable, non-intrusive, lightweight and power-efficient. And it requires no extra hardware and service cost.

- We conduct real driving tests to evaluate the performance of our system. During these tests, we drive regularly or simulate the drunk driving related behaviors. We also vary the position and orientation of mobile phones in the vehicle for the purpose of validation. The results show that our detection system achieves good performance in terms of false negative and false positive.

Paper Organization The rest of the paper is organized as follows. Section II presents related work. We extract the cues of drunk driving in Section III. We present the system design and implementation in Section IV. In Section V, we evaluate our system with real driving tests. In Section VI and VII we discuss our solution and conclude the paper.

\section{RELATED WORK}

There are some existing research on the development and validation of technological tools for driving monitoring. Some of them are known under the name of driver vigilance monitoring, and they focus on monitoring and preventing driver fatigue. Other work focus on real-time driving pattern recognition. In detail, they use various methodologies and techniques described as follows.

Visual observation is an option to detect driver fatigue. In [10], Zhu et al. have used two cameras on dashboard to capture the visual cues of drivers, such as eyelid movement, gaze movement, head movement and facial expression, in order to predict fatigue with a probabilistic model. In [11], Albu et al. have conducted the research in a relatively simpler way.
They claim the sleep onset is the most critical consequence of fatigued driving, separate the issue of sleep onset from the global analysis of the physiological state of fatigue, and take eyes opening and closing as cues of sleep onset. They have used vision-based system to monitor the eyes conditions in order to detect fatigue in driving. In [12], Lee et al. have used two fixed cameras to capture the driver's sight line and the driving lane path for the purpose of driving pattern and status recognition. They calculate the correlation coefficients among them to monitor the driving status and patterns. These methods all need one or more cameras to be installed in the vehicle and just in front of the driver. It will cause certain potential safety hazard to the driver.

Besides visual methods, the interaction at the vehicle-human interface also provides clues to driving information detection. Desai et al., in their work [13], have assumed that the time derivative of force exerted by the driver at the vehicle-human interface, such as pressure on the accelerator pedal, can be used to decide the level of driver alertness. Practically, they have installed a force sensor on the accelerator pedal and collected the exerted force to monitor driver fatigue. In [14], Krajewski et al. have collected steering behavior data and processed them to capture fatigue impaired patterns by using signal processing procedures for feature extraction. They have conducted the experiment with a driving simulator. The automobile manufacturer Saab has proposed an experimental product AlcoKey [15], which collects a breath sample of drivers before they start the vehicle. Then the AlcoKey's radio transmitter sends a signal to the vehicle's electronic control unit to allow it to be started or not based on the alcohol level in the breath sample. These researches use the interactions between human and vehicle to indicate drunk driving. Their systems need to alter the vehicle and be tightly coupled with the auxiliary add-ons, so their compatibility is compromised.

In [16], Heitmann et al. have proposed a series of various technologies, such as head position sensor, eye-gaze system, two pupil-based system and in-seat vibration system, for alertness monitoring and promotion. Based on these technologies, they have used a multi-parametric approach to monitor and prevent driver fatigue. However, their system is very complicated and still stay in the experimental stage, for example the eye-gaze system and pupil-based system are still hard to be integrated into the vehicle.

In [17], Leece et al. have proposed an architecture for driving information system with specific sensors and GPS receiver. They have collected the acceleration and GPS data and used pattern matching to identify and classify driving styles. Their work shows that the acceleration reflects the features of driving pattern. However, they do not only focus on the acceleration signature, but also use GPS data. The devices involved in their system are specific and not conveniently compatible. Also, their system is not realized and still in an early stage of development.

\section{ACCElERATION-BASED DRUnK Driving CUES}

In this section, we analyze the drunk driving related behaviors and extract fundamental cues for drunk driving detection. 


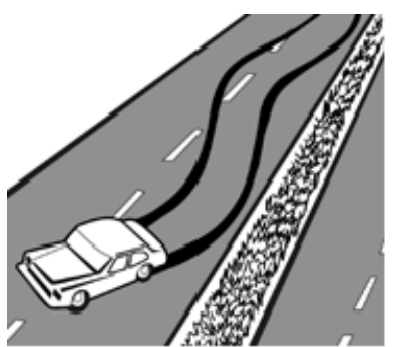

(a)

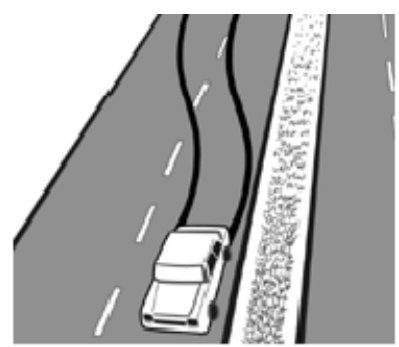

(b)

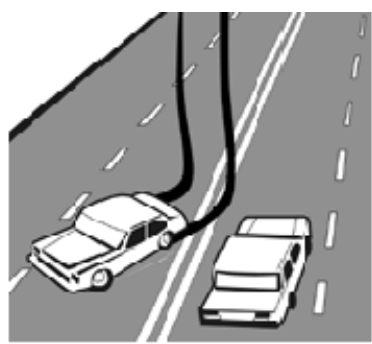

(c)

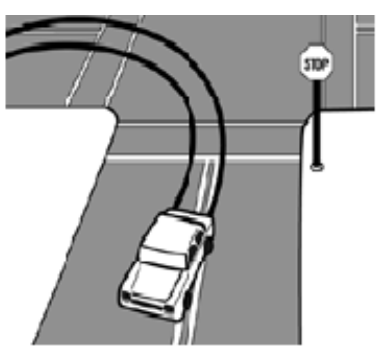

(d)

Fig. 1. Problems in maintaining the lane position : (a) weaving, (b) drifting, (c) swerving, (d) turning with a wide radius [5].

Our analysis is based on the accelerations of vehicles.

In the U.S. NHTSA's study on drunk driving, the researchers have identified cues of typical driving behavior for drunk drivers. Based on their work, we summarize these drunk driving related behaviors into three categories. The first and second category focus on driving behaviors related to vehicle movement itself, such as the movement trace or the movement trend; the third category is about the driving behaviors related to subjective judgment and vigilance of the driver. We present these three categories of behaviors as follows.

- Cues related to lane position maintenance problems: such as weaving, drifting, swerving, and turning abruptly, illegally or with a wide radius.

- Cues related to speed control problems: such as accelerating or decelerating suddenly, braking erratically and stopping inappropriately (e.g. too jerky).

- Cues related to judgment and vigilance problems: such as driving with tires on center or lane marker, driving on the other side of the road, following to closely, driving without headlights at night, and slow response to traffic signals.

According to the U.S. NHTSA's report [5], different categories of cues correspond to different probabilities of drunk driving. Generally, the probability for lane position maintenance problems is $50.75 \%$, for speed control problems $45.70 \%$, for judgment and vigilance problems around $40 \%$. For example, if a driver is observed to be weaving, the probability of drunk driving for him is more than $50 \%$. Some cues, such as swerving and accelerating suddenly, have single-cue probabilities greater than $70 \%$. Furthermore, the probability of drunk driving increases when a driver exhibits more than one of the cues. For example, if the weaving plus any other cue is observed, the probability of drunk driving jumps to at least $65 \%$.

The results suggest that: (1) these driving cues provide relatively strong evidence of drunk driving; (2) the cues related to problems of lane position maintenance and speed control are the main categories, corresponding to higher probabilities of drunk driving, so they can be used as main evidences for drunk driving detection; (3) the probability of drunk driving goes higher while the number of observed cues increases.

For the purpose of developing actively detecting system for drunk driving, we focus on the cues of problems of lane position maintenance and speed control. We map these cues into lateral acceleration and longitudinal acceleration of vehicles.

\section{A. Lateral Acceleration and Lane Position Maintenance}

In general, the lane position maintenance problems result in abnormal curvilinear movements, including weaving, drifting, swerving and turning with a wide radius. They all cause a remarkable change on lateral acceleration. U.S. NHTSA's report gives out the clear illustrations of these situations [5], as shown in Fig. 1.

As illustrated in Fig. 1(a), weaving means the vehicle alternately moves toward one side of the lane and then toward the other. Apparently, the lateral movement is caused by a steering wheel rotation toward one direction and a following steering correction toward the other direction. Similarly, the drifting, swerving and turning with a wide radius have the abnormal lateral movements, as shown in Fig. 1(b)(c)(d).

\section{B. Longitudinal Acceleration and Speed Control in Driving}

A drunk driver often experiences difficulty in keeping an appropriate speed. Abrupt acceleration or deceleration, erratic braking and jerky stop are strong cues to show that the driver is under alcohol impairment. They will all be reflected in the changes of longitudinal acceleration.

We assume that the longitudinal acceleration is positive toward the head of the vehicle. The abrupt acceleration of vehicle will lead to a great increase of longitudinal acceleration (positive values). On the contrary, the abrupt deceleration, erratic braking or jerky stop will cause a great decrease of longitudinal acceleration (negative values).

In summary, the patterns of lateral acceleration and longitudinal acceleration of a vehicle may indicate abnormal lateral movements and abrupt speed variations, which reveal the driver's problems in maintaining lane position and controlling speed. These problems are two main categories of drunk driving related behaviors, and are the strongest cues for detecting drunk driving. Therefore, the acceleration (either lateral or longitudinal) pattern provides fundamental cues for drunk driving detection. Furthermore, multiple rounds of acceleration pattern matching will increase the accuracy of detection, since the probability of drunk driving for a driver increases with more instances of drunk driving related behaviors.

\section{System Design AND IMPLEMENTATION}

In this section, we introduce the design and implementation of our drunk driving detection system. We first present the sys- 


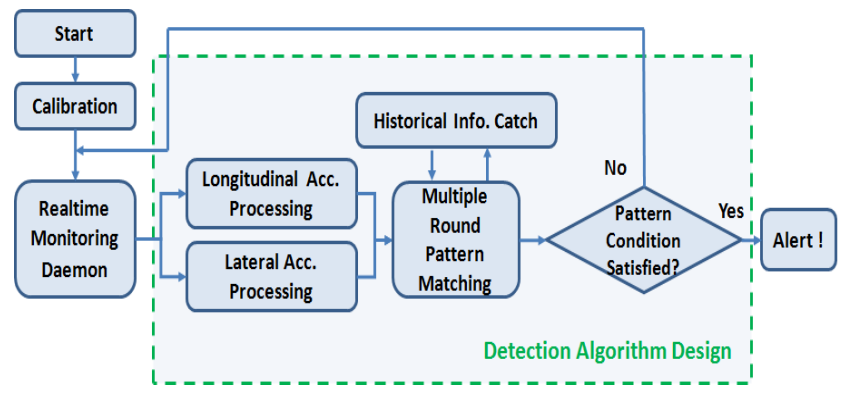

Fig. 2. Working procedure of the drunk driving detection system. The components in the dashed box show the data processing and pattern matching part, reflecting the algorithm design.

tem overview followed by the design of detection algorithms. Then we elaborate on implementation details.

\section{A. System Overview}

The drunk driving detection system is made up of four components, as presented in Fig. 2. They are (1) monitoring daemon module, (2) calibration module, (3) data processing and pattern matching module and (4) alert module. The third module implements the detection algorithm, as marked by a dashed box. Our design is general, not constrained to any particular brand or type of mobile phone. And our design is also power-aware, as hardware such as the screen is only activated when necessary.

The work flow of our drunk driving detection system is also illustrated in Fig. 2. After the system starts manually, a calibration procedure is conducted when the system detects that the phone is located in a moving vehicle. Then the main program launches, working as a background daemon. The daemon monitors the driving behaviors in real time and collects acceleration information. The collected information includes lateral and longitudinal acceleration. They are processed separately, and used as inputs to the multiple round pattern matching process. At the same time, the historical information will be registered. This information is helpful in the following round pattern matching process. If the pattern condition is satisfied, which means a drunk driving is detected, one signal is transmitted to trigger an alert. The phone may alarm to remind the driver or automatically contact the police for help. If the condition is not satisfied, execution returns to the daemon immediately. In the following sections, we will present the details of algorithm design.

\section{B. Design of Algorithm}

We design the detection algorithm based on accelerations, and apply it to the mobile phones equipped with accelerometer and orientation sensor.

The acceleration readings are usually provided by accelerometers in directions of $x$-, $y$-, and $z$-axis, correspondingly represented by $\mathbf{A}_{x}, \mathbf{A}_{y}$ and $\mathbf{A}_{z}$. For generality, we assume that the directions of $x-, y$-, and $z$-axis are decided by the orientation of the phone. As illustrated in Fig. 3 , the $x$-axis has positive direction toward the right side of the device, the $y$-axis has
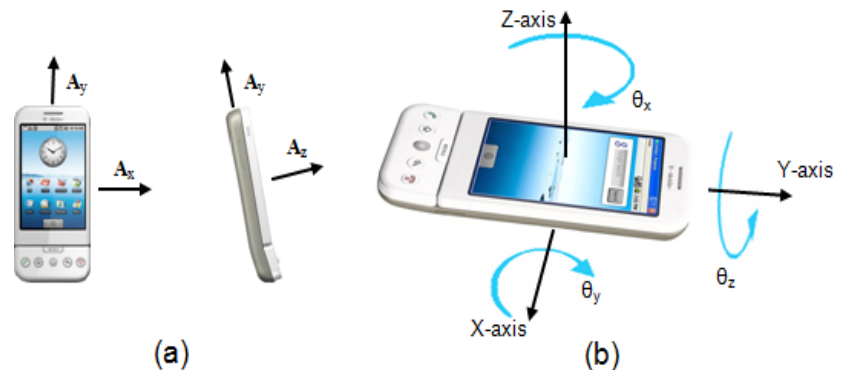

(b)

Fig. 3. (a) Acceleration readings in direction of $x-, y$-, and $z$-axis with regard to the body of the mobile phone. (b) The posture of mobile phone is decided by yaw $\left(\theta_{x}\right)$, pitch $\left(\theta_{y}\right)$ and $\operatorname{roll}\left(\theta_{z}\right)$.

positive direction toward the top of the device and the $z$-axis has positive direction toward the front of the device.

A mobile phone's orientation can be determined by orientation angles, i.e. yaw, pitch, roll values that are denoted as $\theta_{x}$, $\theta_{y}$ and $\theta_{z}$, respectively. The yaw means rotation around the $z$ axis, while pitch and roll represent the rotation around $x$-axis and $y$-axis. They are also shown in Fig. 3. The values of them can be obtained via the orientation sensor.

In real detection process, both the lateral acceleration and the longitudinal acceleration should be based on the vehicle movement direction. The acceleration information of the mobile phone, $\mathbf{A}_{x}, \mathbf{A}_{y}$, should be transformed into the accelerations of the vehicle. In the simplest case, we assume that the mobile phone is laid flat in the vehicle, with the top of phone toward the head of vehicle, so that the accelerations on $x$-axis and $y$-axis represent the lateral and longitudinal accelerations of vehicle, respectively. However, the real situations are more complex. The mobile phone may be laid in the vehicle arbitrarily, neither flat nor heading toward the head of the vehicle. Therefore, we set a calibration procedure to help the system determine what direction is longitudinal. We first obtain the horizontal components of $\mathbf{A}_{x}$ and $\mathbf{A}_{y}$, which are denoted as $\mathbf{A}_{x h}, \mathbf{A}_{y h}$, by Eq. 1 .

$$
\left\{\begin{array}{l}
\mathbf{A}_{x h}=\mathbf{A}_{x} \cos \theta_{z} \\
\mathbf{A}_{y h}=\mathbf{A}_{y} \cos \theta_{y}
\end{array}\right.
$$

The calibration procedure begins to work when the system detects the vehicle starts to move. Its starting movement gives the mobile phone a continuously initial longitudinal acceleration, either forward (to get off directly) or backward (to back off the vehicle first). We denote this acceleration as vector $\mathbf{A}_{I}$. It is much different from that in human movement. Our experiments show that the acceleration keeps above $2.65 \mathrm{~m} / \mathrm{s}^{2}$ for several seconds (at least 3 seconds) during the vehicle's starting movement. During the human movements even in the running, the average acceleration in a time window of 3 seconds is no more than $2 \mathrm{~m} / \mathrm{s}^{2}$. Actually, the most of accelerations in human movements keep below $1 \mathrm{~m} / \mathrm{s}^{2}$. So it is easy for system to detect when the vehicle starts. $\mathbf{A}_{I}$ 's amplitude can be obtained by Eq. 2; while its direction is determined by the direction of $\mathbf{A}_{x h}$ and $\mathbf{A}_{y h}$. 


$$
\left|\mathbf{A}_{I}\right|=\sqrt{\left|\mathbf{A}_{x h}\right|^{2}+\left|\mathbf{A}_{y h}\right|^{2}}
$$

Next, we denote the angle between vector $\mathbf{A}_{x h}$ and $\mathbf{A}_{I}$ as $\alpha$, the angle between vector $\mathbf{A}_{y h}$ and $\mathbf{A}_{I}$ as $\beta$. These two angles can be calculated by Eq. 3 .

$$
\left\{\begin{array}{l}
\alpha=\arccos \left(\mathbf{A}_{x h} /\left|\mathbf{A}_{I}\right|\right) \\
\beta=\arccos \left(\mathbf{A}_{y h} /\left|\mathbf{A}_{I}\right|\right)
\end{array}\right.
$$

Then, the lateral acceleration vector $\mathbf{A}_{\text {lat }}$ and longitudinal acceleration vector $\mathbf{A}_{l o n}$ of the vehicle can be inferred by Eq. 4.

$$
\left\{\begin{array}{l}
\mathbf{A}_{l a t}=\mathbf{A}_{x h} \sin \alpha+\mathbf{A}_{y h} \sin \beta \\
\mathbf{A}_{l o n}=\mathbf{A}_{x h} \cos \alpha+\mathbf{A}_{y h} \cos \beta
\end{array}\right.
$$

The last step of calibration is to determine the correct direction of two vectors $\mathbf{A}_{\text {lat }}$ and $\mathbf{A}_{l o n}$. As we mentioned before, the starting movement of vehicle may be one of two directions. If the vehicle starts to move forward, the vectors we obtained above have the correct directions. Otherwise, the directions of vectors are inverse. Therefore, the system will observe the following moving direction of vehicle after the starting movement. If the direction of following moving differs from the one of starting movement, it will invert the directions of $\mathbf{A}_{\text {lat }}$ and $\mathbf{A}_{\text {lon }}$. Otherwise, these two vectors will remain unchanged.

The determined acceleration vectors $\mathbf{A}_{\text {lat }}$ and $\mathbf{A}_{\text {lon }}$ are used in drunk driving detection, as described in following.

1) Lateral Acceleration Pattern Matching: The lateral acceleration pattern matching is based on the value of $\mathbf{A}_{\text {lat }}$. The pattern which shows remarkable changes of acceleration values reveals the abnormal curvilinear movements of the vehicle. That is when the vehicle is making the curvilinear movement, it has a sudden lateral acceleration toward one side, and then a lateral acceleration toward the other side.

Fig. 4 presents examples of vector $\mathbf{A}_{l a t}$ of a moving vehicle. They are obtained from the acceleration and orientation information collected by the integrated sensors of a mobile phone in that vehicle and calculated through the above equations. Fig. 4 shows the results when the vehicle is operated in different manners by the driver: moving straight, turning normally, weaving and turning with a wide radius. The latter two are considered abnormal curvilinear movements. We can find that in these two scenarios, the values of $\mathbf{A}_{\text {lat }}$ have a great variation from negative to positive.

The pattern matching is to check the variation between the maximum value and the minimum value of $\mathbf{A}_{\text {lat }}$ within a pattern checking time window win lat $_{\text {lat }}$. If the variation exceeds the threshold $T h_{\text {lat }}$, an abnormal curvilinear movement of vehicle is considered happening, showing that the driver has problems in maintaining the lane position. We set the consecutive two checking time windows to have a overlap, in order to avoid missing recognizing pattern.
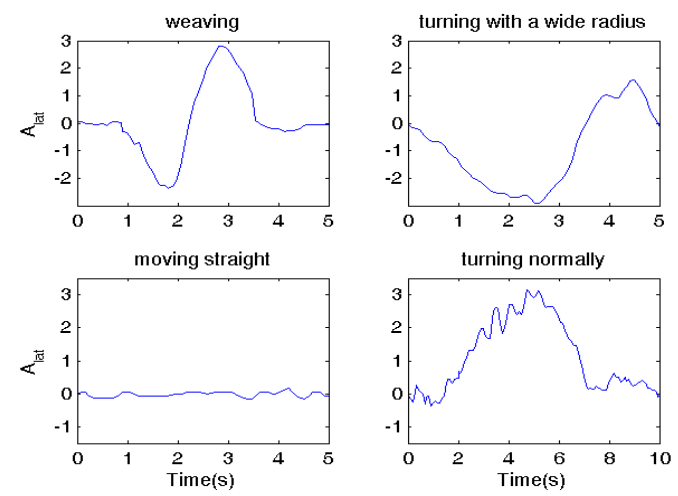

Fig. 4. Examples of $\mathbf{A}_{\text {lat }}$ value of a moving vehicle. We show the results when the vehicle was operated in different manners.

2) Longitudinal Acceleration Pattern Matching: Similarly, the longitudinal acceleration pattern matching is based on the value of $\mathbf{A}_{l o n}$. The problems in speed control, i.e. the vehicle acts abnormally in either accelerating or decelerating direction, result in a large absolute value of $\mathbf{A}_{l o n}$, making a salient convex or concave shape in its graph of curves.

The system keeps checking the maximum or minimum value of $\mathbf{A}_{l o n}$. If the amplitude of value exceeds the threshold $T h_{l o n}$, a speed control problem is considered detected. In reality, the features of accelerating and braking during driving may vary considerably even with the same driver. So that we set different thresholds for positive $\mathbf{A}_{l o n}$ (for accelerating) and negative $\mathbf{A}_{l o n}$ (for decelerating), denoted as $T h_{l o n}^{+}$and $T h_{l o n}^{-}$.

3) Multiple Round Pattern Matching: Multiple round pattern matching will increase the accuracy of drunk driving detection. The cues related to problems of lane position maintenance or problems of speed control provide evidences of drunk driving. The single lateral acceleration pattern matching or longitudinal acceleration pattern matching can indicate these two kinds of problems respectively. Each of the two detection algorithms based on the above two acceleration patterns may achieve a fairly high detection accuracy, above $50 \%$ in most cases, and around $45 \%$ in some cases. Nevertheless, the combination of observed cues from lane position maintenance and speed control problems can provide much stronger evidence for drunk driving. So we use multiple round pattern matching in the detection algorithm design.

We design a simultaneous work flow of data processing for both lateral acceleration and longitudinal acceleration. These two accelerations serve as inputs to the pattern matching module. In pattern matching procedure, the pattern in two directions can be found based on lateral or longitudinal acceleration data. Multiple round means that the matching process continues round after round, and the trigger condition is satisfied when several numbers of pattern are recognized. For this purpose, the historical information catch component is used for catching and storing the previous pattern matching information which can be used in the following round of pattern matching. Currently, we set the triggering condition to be two matching patterns. 


\section{Implementation}

We develop the prototype of the drunk driving detection system on Android G1 phone. The G1 phone provides an accelerometer sensor and an orientation sensor. In the following part, we describe the implementation details of the prototype.

We implement the prototype in Java, with Eclipse and Android 1.6 SDK. It consists of 7 class files, which include 4 Activities, 1 View, 1 Service and 1 Resource. They can be divided into five major components: user interface, system configuration, monitoring daemon, data processing and alert notification. After the system is started, it finishes the configuration automatically. The monitoring daemon keeps running in background as a Service in Android, collecting and recording the readings of sensors. These readings are processed and used to detect drunk driving. In data processing component, according to real situations, the time windows are set to 5 seconds. When drunk driving is detected, the alert notification component works to alarm and remind the driver of dangerous driving or call the police for help.

We compile and build the system project, create and sign the apk file and install it onto G1 phone by ADB tool. The size of the apk application file is about $215 \mathrm{~KB}$. Ultimately, we may create the apk file in release mode, sign it with our release private key and publish it on Android Market, making it available to Android mobile device users for download.

\section{Evaluation}

We evaluate the drunk driving detection prototype with real driving tests. In this section, we first describe how we collect the data. Then we present the system performance in different scenarios and the energy efficiency.

\section{A. Data Collection}

We study drunk driving behaviors in two main categories: problems of lane position maintenance and problems of speed control. Correspondingly, we have collected the data of these two kinds of abnormal driving behaviors in real driving simulations. The data include lateral and longitudinal accelerations of the vehicle, reflecting its features of movement in these two directions. We have also collected the data of regular driving in different environments. In the experiments, the mobile phone was laid in the vehicle. In most cases, it did not slide, since the friction between the body of phone and the surface inside the vehicle was strong enough to hold the phone in the same position during driving. But we still consider the situations in which the phone was put on a slippery surface, and might slide in driving. We have conducted further tests to see how our detection system applies to this special case. We report the experimental data in the follow section. In general, we separate the driving behavior data collected into two sets, one for training and the other for evaluation.

In the experiments, we drove a 2002 Ford Taurus car, having a G1 phone with our drunk driving detection system in the car. Since the system finishes the calibration procedure automatically, there is no requirement for the position and orientation of the phone. We arbitrarily laid the phone in the
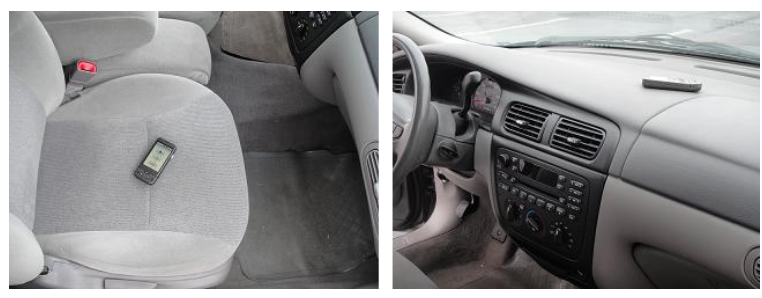

Fig. 5. The G1 phone is put on the front seat or the dashboard of the vehicle during drunk driving detection.

car, e.g., on the front right seat, on the back seat, on the dashboard, etc., as shown in Fig. 5. Then we drove the car with different driving behaviors and in different environments. The main categories of drunk driving related behaviors and regular driving behaviors were both tested. And general driving locations, such as in alley, on highway, in campus and etc., are considered.

In total, we have obtained 72 sets of data for drunk driving related behaviors, including weaving, swerving, turning with a wide radius and erratically changing speed (accelerating or decelerating); and we also collected 22 sets of data for regular driving, each of these drivings lasts for 5 to 10 minutes.

\section{B. Detection Performance}

We study the performance of detecting drunk driving related behaviors, since drunk driving can be directly inferred by the accurate detection of these abnormal driving behaviors. We measure the detection performance in terms of false negative (FN) and false positive (FP). False negative happens when drunk driving related behaviors show up but the device misses them. False positive happens when the device reports drunk driving related behaviors but the vehicle is actually under regular driving conditions. In general, the lower the both FN and FP are, the better the performance is.

1) Performance Description: We first determine all the parameter values in the algorithm. We choose win lat to be 5 seconds, because we find all the patterns for drunk driving related behaviors happen in such a time period according to our test data. We may obtain thresholds for the determination of drunk driving related behaviors in our detection algorithm from the training data. When determining the threshold values, we consider the tradeoff between FN and FP, because any decrease in FN will be accompanied by an increase in FP. With different threshold values, we can achieve different balances between FN and FP, aiming at low FN and reasonable FP. We choose one threshold setting, described as follows, that achieves the best balance between FN and FP with our training data. For detection of the abnormal curvilinear movement in drunk driving related behaviors, we set the threshold $T h_{\text {lat }}$ to 4 . That means a upper bound of variation in values of $\mathbf{A}_{\text {lat }}$. If the variation of $\mathbf{A}_{\text {lat }}$ values exceeds that threshold, the vehicle is very likely to be making an abnormal curvilinear movement; otherwise, its lateral acceleration would not have such a great change in a small time period. For detection of problems of 


\begin{tabular}{|c|c|c|}
\hline & Abnormal Curvilinear Movements & Problems of Speed Control \\
\hline FN Rate (\%) & 0 & 0 \\
\hline FP Rate (\%) & 0.49 & 2.39 \\
\hline $\begin{array}{c}\text { FN Rate (\%) } \\
\text { (Phone Slides) }\end{array}$ & 14.28 & 0 \\
\hline $\begin{array}{c}\text { FP Rate (\%) } \\
\text { (Phone Slides) }\end{array}$ & 1.09 & 2.72 \\
\hline
\end{tabular}

Fig. 6. Experimental performance from real driving test. We make a comparison between the cases in that the phone slides or not during driving.

speed, we set the threshold $T h_{\text {lon }}^{-}$to 2.7 and $T h_{\text {lon }}^{+}$to 2.4 . Those are the upper bounds of speed changes in accelerating and decelerating. According to our test, one vehicle in regular driving does not speed up or slow down too fast, and the positive or negative acceleration values will not exceed the thresholds during all driving.

In our experiment, we have tested the detection performance in abnormal curvilinear movement and problem in maintaining speed, which are the two main categories of drunk driving related behaviors. Fig. 6 shows the experimental results obtained from testing data. In detection of abnormal curvilinear movement, the FN rate is $0 \%$, and the FP rate is $0.49 \%$. In detection of speed control problem, the FN rate is also $0 \%$, and the FP rate is $2.39 \%$. The result shows that the detection algorithm in our system performs very well. It can detect all the drunk driving related behaviors, such as abnormal curvilinear movements and problems of speed control, while the false alarm for regular driving behavior is limited to a reasonable range. Especially, the detection for abnormal curvilinear movements has the best performance.

2) Performance in Special Case: As we mentioned before, in a few special cases, the phone may slide in the vehicle during driving. Apparently, if so, the phone's accelerations in lateral direction or longitudinal direction or both will differ from the accelerations of the vehicle at the very moment of sliding; the phone's position and orientation may also change. Therefore the driving behavior detection based on vehicle accelerations might be affected.

For the purpose of testing the detection in these cases, we gather all the data when the phone slides during drunk driving detection. The data include both drunk driving related behaviors and regular driving behaviors. We test the detection performance by these data with the same threshold set before. Fig. 6 shows the results. In these special cases, the FN rate for detecting abnormal curvilinear movement is $14.28 \%$, and the FP rate is $1.09 \%$; the FN rate for speed control problem is $0 \%$, and the FP rate is $2.72 \%$.

The result shows that the slide of mobile phone has obvious impacts on the detection accuracy, especially for detecting abnormal curvilinear movements. Because if the phone slides when the vehicle is making curvilinear movements, the phone is very likely to rotate around an axis. So the lateral acceleration of the phone and that of the vehicle will have a quite large difference. In other words, it is hard to use the lateral acceleration of the phone to determine the moving trend of vehicle in lateral

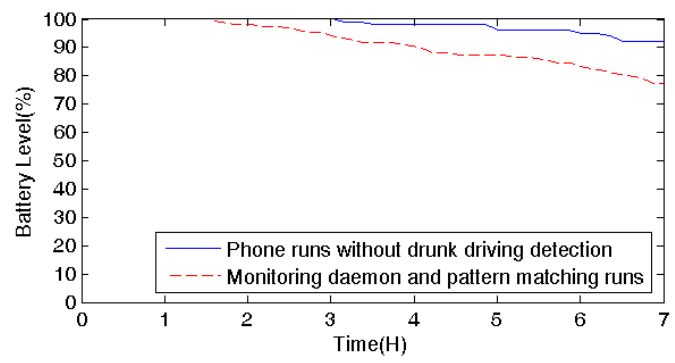

Fig. 7. Power consumption curves in different scenarios.

direction.

To solve the problems caused by position and orientation changes when phone slides during driving, we may add an additional calibration procedure in the detection algorithm to improve the detection performance in this special case.

\section{Energy Efficiency}

To test power consumption of the detection system, we fully charge the G1 phone and then monitor the power states continuously for 7 hours in different scenarios: 1) the G1 phone runs without drunk driving detection; 2) the monitoring daemon of system keeps running, sensing and recording acceleration and orientation, and do the pattern matching on the monitoring results. Fig. 7 illustrates the two curves of battery level states versus time during this time period of 7 hours. If the drunk driving detection system keeps running until the battery power is exhausted, it will last a little more than 34 hours.

\section{DISCUSSION}

In this section, we discuss some untouched issues in the general design and implementation.

1) GPS: Since all our detection approaches rely on driving patterns and vehicle movements, it is intuitive to think of GPS as a helpful auxiliary. With GPS, it is easy to delineate the vehicle movement trace and compare it with the road directions. Some simple graphic processing techniques may be sufficient to pinpoint any abnormal curves present in the trace. Moreover, GPS can provide us with another valuable parameter, the speed of the vehicle. Though we can infer the speed based on the integral of accelerations, cumulative error will make the inference meaningless after a period of several minutes. With this speed information, we can identify dangerous driving pattern with finer granularity. For example, if the vehicle runs at a very high speed, a small lateral acceleration is enough to make it drift or swerve with large dangerous S-curves. So the acceleration threshold of alerting should be dynamically adjusted according to the speed readings. Another benefit from the speed information is that we are able to calculate the radius of curvature of the vehicle moving path with Radius $=$ speed $^{2} /$ acceleration $_{\text {lateral }}$. The radius can be a very accurate indication whether the driver is making irregular and consecutive sharp turns.

Though the integration of GPS information is worth exploring and is one direction of our future work, we argue 
here that GPS is not the best choice for our current solution. First, while accelerometers and orientation sensors, which we have adopted in our solution, are cheap and available on many mobile phones, GPS is a fairly sophisticated functionality that is only present on high-end smartphones. So the generality of our detection mechanism is compromised if it depends heavily on GPS. Another major concern about GPS is its localization accuracy. It is common sense that a localization error at the magnitude of several meters can take place with GPS [18]. As we can see from the NHTSA cue patterns, a single abnormal vehicle maneuver normally happens within a distance of 10 to 20 meters. So the GPS localization error may cause great impact on the detection accuracy, if we consider the already achieved accuracy at around $100 \%$ with accelerometer and orientation sensors alone. On the other hand, while accelerometers and orientation sensors are mainly mechanical sensors and consume little energy, GPS, if turned on all the time, is highly energy consuming, as it constantly receives signals from satellites and performs considerable amount of computations.

2) Camera: Most mobile phones are equipped with a camera with tolerable quality, e.g., 2 Megapixel resolution probably with other functionalities such as auto focus and exposure compensation. So a mobile phone is usually capable of acquiring visual information. It can be a great help to remedy some weaknesses of the solution presented above. For example, cameras can be used to follow drivers' sight line and capture distinctive road signs or marks to help analyze driving patterns [10] [12]. However, several factors prevent us from adopting this option. First, in order to capture useful visual information, there are many requirements on the position and posture of the phone. A mobile phone must be meticulously set in a vehicle to face the front at an appropriate angle. A little position shift or sliding can totally ruin the camera view [19]. So it is not feasible even to ask normal drivers to maintain an accurate position of his mobile phone, not to say a drunk driver. Second, the algorithm behind the visual identification must be very complicated to accommodate various and changeable traffic conditions. A lot of intensive computations must be performed in a real time fashion and, hence, a potential high false positive/negative rate is possible given the limited computation capability of mobile phones. Third, camera operations and intensive image processing consume a lot of energy, particularly if the visual monitoring is conducted with a relatively high frequency. So in short, we think it is impractical at present to include camera and visual monitoring into our solution. However, it is a potential extension to our system with the fast development of mobile phone hardware in the future.

\section{CONCLUSION}

In this paper, we present a highly efficient mobile phone based drunk driving detection system. The mobile phone, which is placed in the vehicle, collects and analyzes the data from its accelerometer and orientation sensor to detect any abnormal or dangerous driving maneuvers typically related to driving under alcohol influence. Experiments show that our solution sees very low false negative and false positive rates, as well as tolerable energy consumption. In our future work, we plan to improve our detection system by integrating all available sensing data on a mobile phone, e.g., GPS data and camera image.

\section{ACKNOWLEDGMENT}

This work was supported in part by the US National Science Foundation (NSF) under grants No. CNS-0916584, CAREER Award CCF-0546668, and the Army Research Office (ARO) under grant No. AMSRD-ACC-R 50521-CI. Any opinions, findings, conclusions, and recommendations in this paper are those of the authors and do not necessarily reflect the views of the funding agencies.

\section{REFERENCES}

[1] J. Faber, "Detection of Different Levels of Vigilance by EEG Pseudo Spectra", in Neural Network World, 14(3-4), pp. 285-290, 2004.

[2] U.S. NHTSA, "Traffic Safety", http://www-nrd.nhtsa.dot.gov/Pubs/ 811172.pdf

[3] U.S. CDC, "Mobile Vehicle Safety-Impaired Driving", http://www.cdc. gov/MotorVehicleSafety/Impaired_Driving/impaired-drv_factsheet.html

[4] M. H. Lee, M. J. Mello and S. Reinert, "Emergency Department Charges for Evaluating Minimally Injured Alcohol-Impaired Drivers", in Annals of Emergency Medicine, Vol. 54, No. 4, pp. 593-599, Oct. 2009.

[5] U.S. NHTSA, "The Visual Detection of DWI Motorists", http://www. nhtsa.dot.gov/people/injury/alcohol/dwi/dwihtml/index.htm

[6] P. Resende, "Smartphone Competition Heats Up With Many Choices", NewsFactor Network, Sept. 2008, http://www. newsfactor.com/story.xhtml?story_id=61671.

[7] M. Boran, "How the Mobile Social Web Will Make 2009 a Smart Phone Odyssey", http://www.independent.ie/business/ technology/how-the-mobile-social-web-will-make-2009-a-smart-phoneodyssey-1609944.html.

[8] G. Mies, "Six New Phones from AT\&T: Have a Look", PC World, Mar. 30, 2009, http://pcworld.about.com/od/ smartphones/SixNew-Phones-from-AT-amp-T.htm

[9] "New Smartphone Family Wireless Plan from Cincinnati Bell", http://wirelessplansinformation.blogspot.com/2008/10/new-smartphonefamily-wireless-plan.html

[10] Z. Zhu and Q. Ji, "Real Time and Non-intrusive Driver Fatigue Monitoring", in The 7th International IEEE Conference on Intelligent Transportation Systems, pp. 657-662, Oct. 2004.

[11] A. B. Albu, B. Widsten, T. Wang, J. Lan and J. Mah, "A Computer Vision-Based System for Real-Time Detection of Sleep Onset in Fatigued Drivers", in 2008 IEEE Intelligent Vehicles Symposium, pp. 25-30, June 2008.

[12] J. Lee, J. Li, L. Liu and C. Chen, "A Novel Driving Pattern Recognition and Status Monitoring System", in First pacific rim symposium, PSIVT 2006, pp. 504-512, December 2006.

[13] A. V. Desai and M. A. Haque, "Vigilance Monitoring for Operator Safety: A Simulation Study on Highway Driving”, in Journal of Safety Research, Vol. 37, No. 2, pp. 139-147, 2006.

[14] J. Krajewski, D. Sommer, U. Trutschel, D. Edwards and M. Golz, "Steering Wheel Behavior Based Estimation of Fatigue", in Proceedings of the Fifth International Driving Symposium on Human Factors in Driver Assessment, Training and Vehicle Design, pp. 118-124.

[15] "Saab AlcoKey Helps Drivers", http://www.saabnet.com/tsn/press/061013a.html.

[16] A. Heitmann, R. Cuttkuhn, A. Aguirre, U. Trutschel and M. Moore-Ede, "Technologies for The Monitoring and Prevention of Driver Fatigue", in Proceedings of the Fifth International Driving Symposium on Human Factors in Driver Assessment, Training and Vehicle Design, pp. 81-86.

[17] V. D. Lecce and M. Calabrese, "Experimental System to Support RealTime Driving Pattern Recognition", in Advanced Intelligent Computing Theories and Applications With Aspects of Artificial Intelligence Annals of Emergency Medicine, pp. 1192-1199, 2008.

[18] Wikipedia, "GPS" entry, http://en.wikipedia.org/wiki/Global_Positioning System

[19] M. Wilczkowiak, E. Boyer, and P. Sturm, "Camera Calibration and 3D Reconstruction from Single Images Using Parallelepipeds", in Proceedings of ICCV, pp. 142-148, 2001. 